Title : will be set by the publisher

Editors : will be set by the publisher

EAS Publications Series, Vol. ?, 2018

\title{
INFRARED PROPERTIES OF AGB STARS: FROM EXISTING DATABASES TO ANTARCTIC SURVEYS.
}

\author{
R. Guandalini ${ }^{1}$ and M. Busso ${ }^{1}$
}

\begin{abstract}
We present here a study of the Infrared properties of Asymptotic Giant Branch stars (hereafter AGB) based on existing databases, mainly from space-borne experiments. Preliminary results about $\mathrm{C}$ and $\mathrm{S}$ stars are discussed, focusing on the topics for which future Infrared surveys from Antarctica will be crucial. This kind of surveys will help in making more quantitative our knowledge of the last evolutionary stages of low mass stars, especially for what concerns luminosities and mass loss.
\end{abstract}

\section{Introduction}

' Towards the end of their life, stars of low and intermediate mass $\left(M<8 M_{\odot}\right)$ - evolve along the Asymptotic Giant Branch (AGB) phase [see Busso et al. (1999) , and references herein for more details]. In this stage they experience extensive phenomena of mass loss that affect deeply their evolution. Sedlmayr (1994) shows that stellar winds are also fundamental for the enrichment of the Interstellar Medium that is replenished by these stars with about $70 \%$ of all the matter returned after stellar evolution. Moreover, AGB stars provide the starting conditions for the formation of planetary nebulae.

The radiative emission of the cool dust in the infrared (IR) normally dominates the energy distribution of AGB stars (particularly for the most evolved ones) and this fact is mainly due to their strong stellar winds. Until recently the bolometric magnitude of the most evolved AGB stars was difficult to derive, due to insufficient photometric coverage of the mid-IR range of the electromagnetic spectrum (the importance of mid-IR observations for AGB stars is clearly shown in Guandalini et al. (2006), Figure 1). The availability of large IR databases from space-borne telescopes like ISO, IRTS, MSX has substantially improved this situation. At the same time, Hipparcos distances for AGB stars have been corrected from various

\footnotetext{
${ }^{1}$ Dipartimento di Fisica, Università di Perugia, Via A. Pascoli, 06123 Perugia, Italy; e-mail: guandalini@fisica.unipg.it
}

(C) EDP Sciences 2018

DOI: (will be inserted later) 
Title : will be set by the publisher

biases in works like the one from Bergeat \& Chevallier (2005) and the periodluminosity relations found for Miras have been drastically improved as shown in Whitelock et al. (2006). The study of fundamental physical parameters of these stars (like luminosity, IR colors and mass loss) can now be be performed in a rather quantitative way.

However, IR space-borne observations of AGB stars present some disadvantages. In particular, the duration of the operational period of the telescope is quite limited, observations with a long time of integration are difficult and AGB stars are generally observed at a single epoch. All these facts hinder our understanding of several basic physical parameters, which are fundamental in the study of AGB stars. In this respect a complementary role in solving these problems could be played by ground-based observations at IR wavelengths, especially from Antarctica.

The Antarctic Plateau (in particular Dome C) presents the best conditions available on Earth from the point of view of infrared observations as shown in several other contributions from this conference: therefore, Antarctica is the best place where ground-based observations in the (mid-)IR can be performed. An Antarctic IR Observatory might be crucial for clarifying the final stages of stellar evolution by:

- observing properties of evolved stars in the Magellanic Clouds, at known distance and metallicity different from the Milky Way;

- doing the same for the Galactic Center, where the metal blend is different and the extreme O-enhancement prevents the formation of $\mathrm{C}$ stars as shown in Uttenthaler et al. (2006);

- looking for mass loss calibrations in the IR.

Our efforts are addressed to the preparation for IR observations of AGB stars from Antarctica through the IRAIT telescope, presented by Tosti et al. (2007) in this conference. With this aim we are trying to understand which kind of observations from Antarctica is the best and most promising from the point of view of AGB stars. In Guandalini et al. (2006), (2007) we are making for these sources an overview of the IR data available from existing catalogues that will be completed in future works. In this note we address some interesting issues concerning AGB stellar variability from an IR point of view, and the relevance of Antarctic observations for them.

\section{Infrared Variability}

AGB stars present strong variability at optical wavelengths and are roughly divided into three subclasses according to their variability type: Miras, Semiregulars and Irregulars. Effects due to variability at IR wavelengths are expected to be less relevant when compared with the optical ones.

Figures 1 1 and2 present the available information on the IR SEDs for two groups of AGB sources that are discussed in detail in Busso et al. (2006). Figure 1 shows 


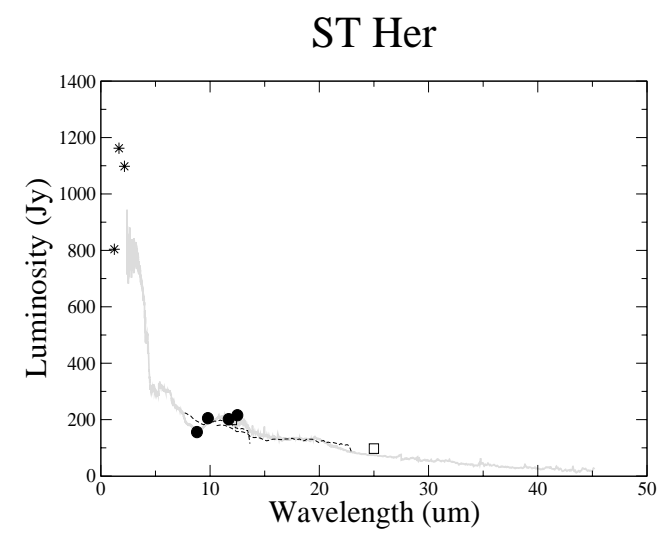

HD 56126

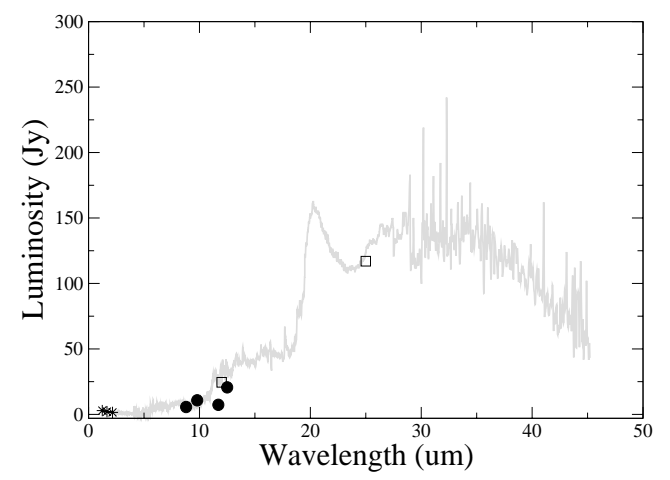

RY Dra

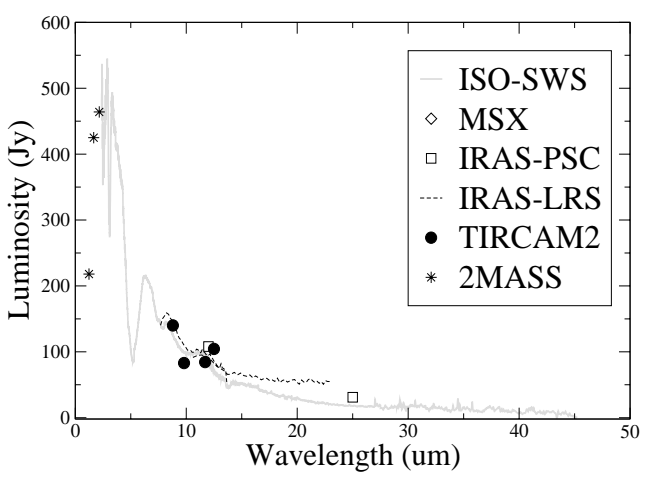

Red Rectangle

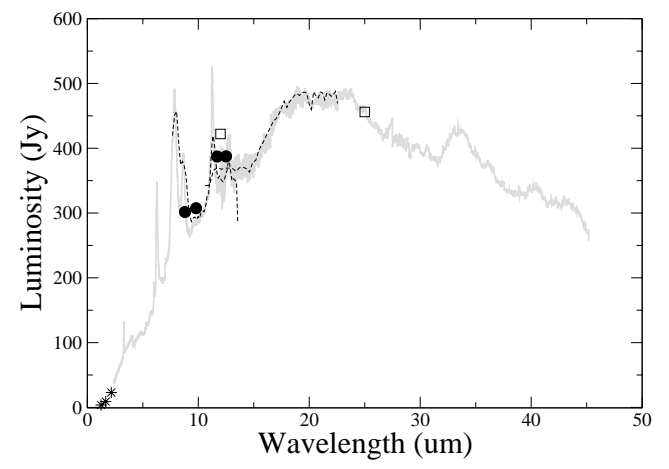

Fig. 1. The Spectral Energy Distributions (SEDs) of a few sources, as available from IRAS PSC, IRAS LRS, ISO-SWS, MSX, TIRCAM2 and 2MASS. SEDs with maximum emission in near-IR, as well as with maximum emission longward of $20 \mu \mathrm{m}$ all show a constant flux in mid-IR.

distributions that share the property of being non-variable at IR wavelengths over an elapse of time of almost 20 years. They include Semiregular sources with minimal IR excess, in which the SED is peaked in near-IR. They also include evolved (post-AGB) objects in which the maximum emission is at very long wavelengths (from 20 to more than $40 \mu \mathrm{m}$ ). Instead, Figure 2 shows a group of Mira variables, in which the emission peaks near $10 \mu \mathrm{m}$ : they present the common property of a long-term mid-IR variability that seems to be restricted to this special class of sources. Moreover, Figure 3 shows two of the few available AGB sources observed several times by ISO-SWS and confirms the same behavior: Mira variables present an IR variability even over a relatively short time interval.

There is not yet an agreement on the origin and properties of this phenomenon. 
Title : will be set by the publisher
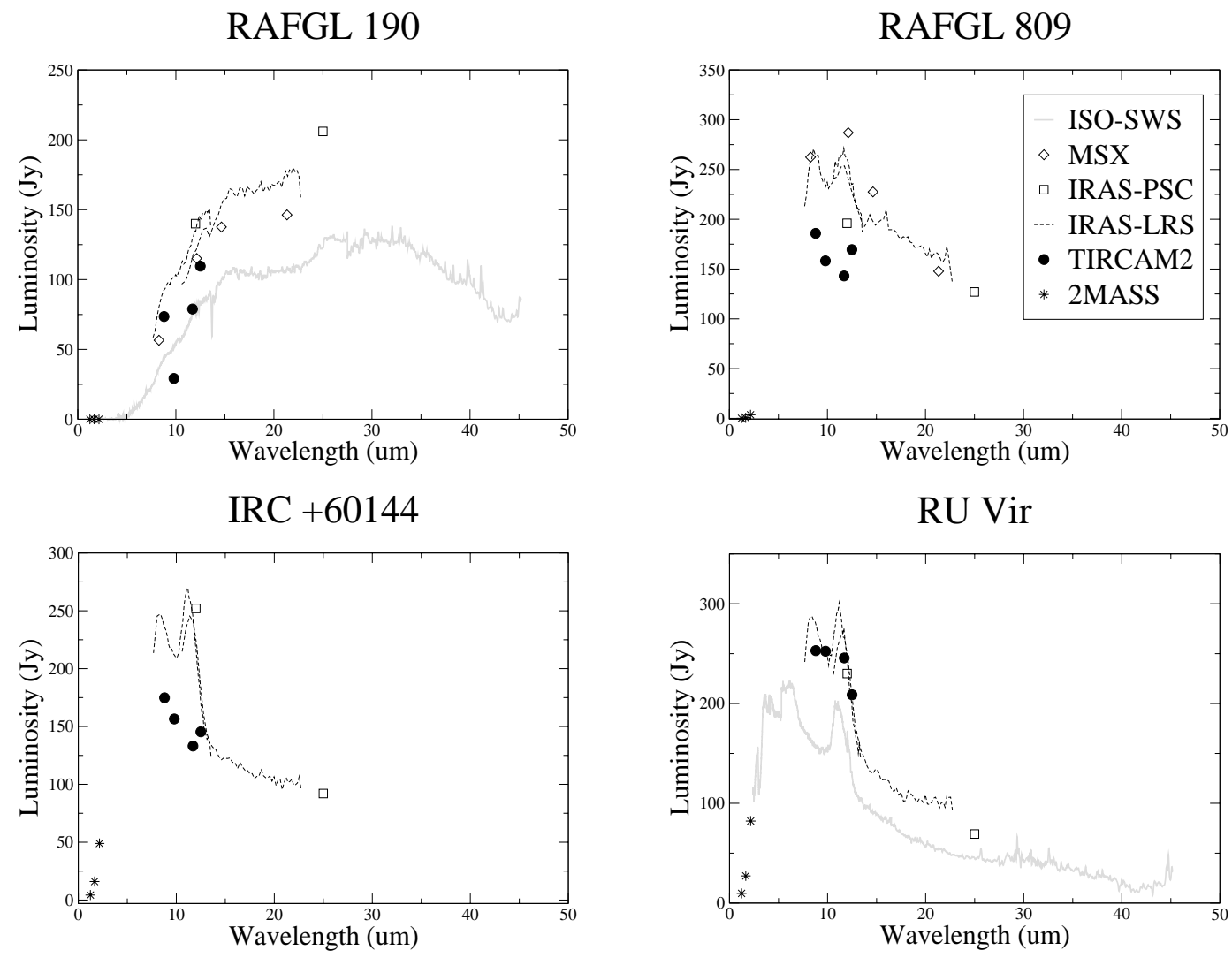

Fig. 2. SEDs of sources that show significant variability over the time elapsed from the IRAS to the TIRCAM2 observations. Data available from IRAS PSC, IRAS LRS, ISOSWS, MSX, TIRCAM2 and 2MASS are included. Only sources with maximum emission in the range $8-20 \mu \mathrm{m}$ appear to be variable.

It needs to be examined in more detail to understand its nature. It could be perhaps a variability induced by shock waves caused by dynamic events in the photosphere or by magneto-hydrodynamical modes (and magnetic storms). Otherwise, it could be a "simpler" mid-IR variability, originating in the emission of the circumstellar envelopes and caused by modulations in the efficiency of dust formation. This would be expected to be more typical of the Mira sources; Semiregulars have thinner circumstellar envelopes, while Post-AGB stars should have detached shells not strongly influenced by the variability of the central star.

The best way to examine this variability is that of observing AGB stars at different epochs also in mid-IR and this task can be performed by a groundbased telescope placed in Antarctica. Moreover, simultaneous observations in the 

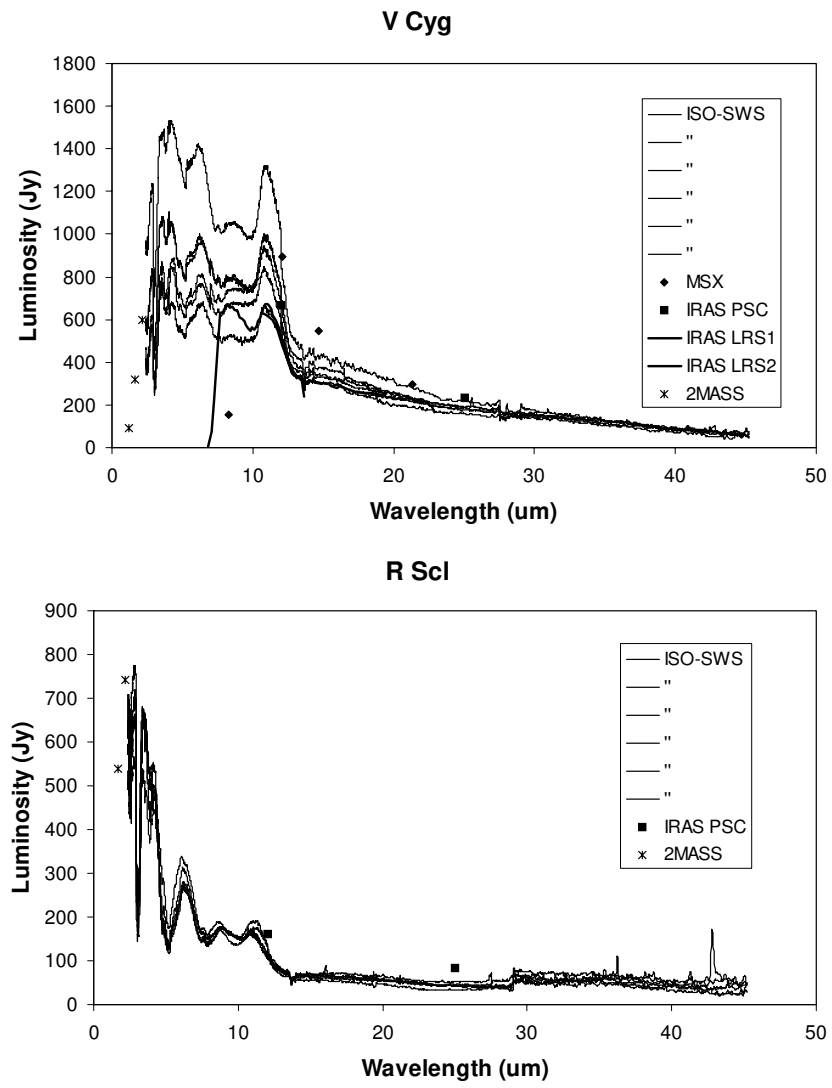

Fig. 3. SEDs of two sources observed several times with ISO-SWS. Only the source with maximum emission in the $10 \mu \mathrm{m}$ region appears to be variable.

near- and mid-IR and correlations with optical variability could be fundamental to understand these phenomena.

\section{Conclusions: Why Surveys from Dome C}

Important IR studies for AGB stars can be made in the best way from groundbased locations like Dome $\mathrm{C}$ and Antarctica. The study of the main features regarding IR variability (and also optical variability) is one of them. It can be fulfilled with, surveys through wide field (in the future) or small area (IRAIT) imaging of interesting stellar systems, multiple observations of chosen AGB sources at different epochs and wavelengths and observation of AGB sources from Magellanic Clouds, whose distance is well-estimated.

In this way we could obtain: 
- light curves and therefore good knowledge of variability;

- an accurate study of the luminosity variations over a wide region of the electromagnetic spectrum, including optical and IR;

- finally, a reliable comparison between observations and models of AGB stars.

\section{References}

Bergeat, J., \& Chevallier, L. 2005, A\&A, 429, 235

Busso, M., Gallino, R., \& Wasserburg, G. J. 1999, ARA\&A, 37, 239

Busso, M., Guandalini, R., Persi, P., Corcione, L., \& Ferrari-Toniolo, M. 2006, AJ, submitted

Guandalini, R., Busso, M., Ciprini, S., Silvestro, G., \& Persi, P. 2006, A\&A, 445, 1069

Guandalini, R., Busso, M., \& Cardinali, M. 2007, in preparation

Sedlmayr, E. 1994, Lecture Notes in Physics, 428, 163

Straniero, O., Domínguez, I., Cristallo, S., \& Gallino, R. 2003, PASA 20, 389

Tosti, G. et al. 2007, this conference

Uttenthaler, S., Hron, J., Lebzelter, T., Busso, M., Schultheis, M., \& Kaeufl, H. U. 2006, astro-ph/0610500

Whitelock, P. A., Feast, M. W., Marang, F., \& Groenewegen, M. A. T. 2006, MNRAS, 369, 751 